\title{
Functioning at 6 months post stroke following discharge from inpatient rehabilitation
}

\author{
Susan Rouillard, Willy De Weerdt, Liesbet De Wit, Jennifer Jelsma
}

Objectives. To determine activity limitations, participation restrictions, health-related quality of life and caregiver strain in community-dwelling stroke survivors discharged from an intensive inpatient rehabilitation programme at 6 months post stroke.

Methods. Fifty-one consecutive stroke patients admitted to a Western Cape rehabilitation centre were included. Communitydwelling participants $(N=46)$ at 6 months post stroke were assessed using the Modified Rankin Scale (MRS), Barthel index (BI), Nottingham extended activities of daily living (NEADL) scale, Euroqol (EQ-5D) instrument and caregiver strain index (CSI).

Results. Most participants (73.9\%) were independent in activities of daily living or had minimal disability (BI 75 - 100). However, according to the NEADL, many participants were not independent in housework (60.9\%), food preparation (52.2\%), shopping (80.4\%) and public transport use (65.2\%), implying the need for caregiver assistance. According to the MRS, $29 \%$ of participants were severely disabled, requiring caregiver assistance for basic needs, and $20 \%$ could not be left alone. Feelings of anxiety or depression were felt in 50\% of participants and 59\% reported pain or discomfort, according to the EQ-5D. High levels of caregiver strain were reported in $56 \%$ of caregivers (CSI). Follow-up after discharge was reported in few participants.

Conclusions. Consideration should be given to support for stroke survivors and caregivers after discharge and whether targeted programmes can improve specific aspects of functioning, such as community mobility.

S Afr Med J 2012;102(6):545-548.
Stroke is a major cause of death in South Africa (SA) and the incidence is increasing. ${ }^{1}$ Many patients survive the initial event but are left with disability and face the challenge of reintegrating into residential and community living. ${ }^{2,3}$

Little information is available on stroke survivor functioning in SA, particularly at the level of activity limitation (difficulties in task execution) and participation restriction (problems experienced in involvement in life situations). ${ }^{4} \mathrm{~A}$ study in a deprived rural community reported a higher prevalence of stroke survivor disability than expected on the basis of international studies. ${ }^{5}$ In another study of patients discharged from a remote rural hospital, $60 \%$ had a Modified Rankin Scale (MRS) score of 0 - 3 at 3 months and participation in work, social activities, housework and sport was reduced. ${ }^{6}$ Patients receiving rehabilitation at community healthcare centres (CHCC) in the Western Cape experienced problems with extended activities of daily living (ADL) and community integration. ${ }^{7}$ These studies refer to stroke cohorts receiving little rehabilitation input. There are few data on the outcomes of patients who are able to access intensive inpatient rehabilitation in SA. ${ }^{8}$

We aimed to determine the activity limitations and participation restrictions, health-related quality of life and caregiver strain in survivors discharged from an inpatient rehabilitation facility at 6 months post stroke.

Division of Physiotherapy, Department of Health and Rehabilitation Sciences, University of Cape Town

Susan Rouillard, MSc (Physio)

Jennifer Jelsma, $\mathrm{PhD}$

Department of Rehabilitation Sciences, Faculty of Kinesiology and Rehabilitation Sciences, Katholieke Universiteit Leuven

Willy De Weerdt, $\mathrm{PhD}$

Liesbet De Wit, $\mathrm{PhD}$

\section{Methods}

This study was part of a larger, longitudinal and descriptive study that drew from the methodology of the European Collaborative Evaluation of Rehabilitation in Stroke across Europe (CERISE) project. CERISE included a multi-centre comparison between stroke rehabilitation units in Europe. ${ }^{9}$ In our study, consecutive stroke patients were recruited between June 2005 and March 2006 from the Western Cape Rehabilitation Centre (WCRC), a regional specialist centre offering inpatient interdisciplinary rehabilitation. Inclusion criteria were: (i) first-ever stroke according to the World Health Organization definition of 'rapidly developing clinical signs of focal (or global) disturbance of cerebral function, with symptoms lasting 24 hours or leading to death, with no apparent cause other than vascular origin'; ${ }^{10}$ (ii) admission to the WCRC within 3 months post stroke; (iii) 18 - 85 years of age; (iv) living within $50 \mathrm{~km}$ of the WCRC. Patients were excluded if they had pre-stroke neurological conditions.

Outcome measures in terms of stroke disability were: ADL independence according to the Barthel index (BI); ${ }^{11}$ abilities for community living according to the Nottingham extended activities of daily living (NEADL) scale, ${ }^{7,9,12}$ and global disability according to the Modified Rankin Scale (MRS) structured interview. ${ }^{13,14}$ Responses to individual questions on the MRS structured interview were used to describe perceived changes in usual duties and activities - including work, family responsibilities, social and leisure activities - and relationships with family and friends. Health-related quality of life was assessed with the Euroqol (EQ-5D) instrument, ${ }^{15}$ and the caregiver strain index $(\mathrm{CSI})^{16}$ was used to determine levels of caregiver strain.

The NEADL, CSI and MRS were translated into Afrikaans and Xhosa and back-translated, before a consensus version was produced. Reliability testing of the translated instruments was conducted on a small group of 1 - 6 patients using a test-retest methodology; although no statistical analysis was possible (small sample size), no major cultural or environmental issues were identified that invalidated their use. Patients living in the community were assessed at 6 months post stroke using the BI, NEADL, EQ-5D, CSI and MRS. Participants with severe cognitive or language deficits were excluded from EQ-5D analysis. Proxies were not asked to respond on the respondents' behalf to avoid introducing bias. ${ }^{17}$ 
Onset to admission interval and length of stay in the rehabilitation centre were documented, as well as post-discharge contact with health services for follow-up (occupational, physio-, speech and language therapy, social work, and medical or nursing assistance).

Assessments were conducted by the researcher (SR) in English or Afrikaans, and in isiXhosa with the assistance of a translator. Unmet clinical needs, identified by the researcher, were referred to the appropriate services. The Ethics Committee of the University of Cape Town approved the study. Descriptive statistics were used to represent the data.

\section{Results}

Eligibility criteria were met by 59 patients; 1 refused consent, and 7 could not give informed consent due to cognitive or language deficits

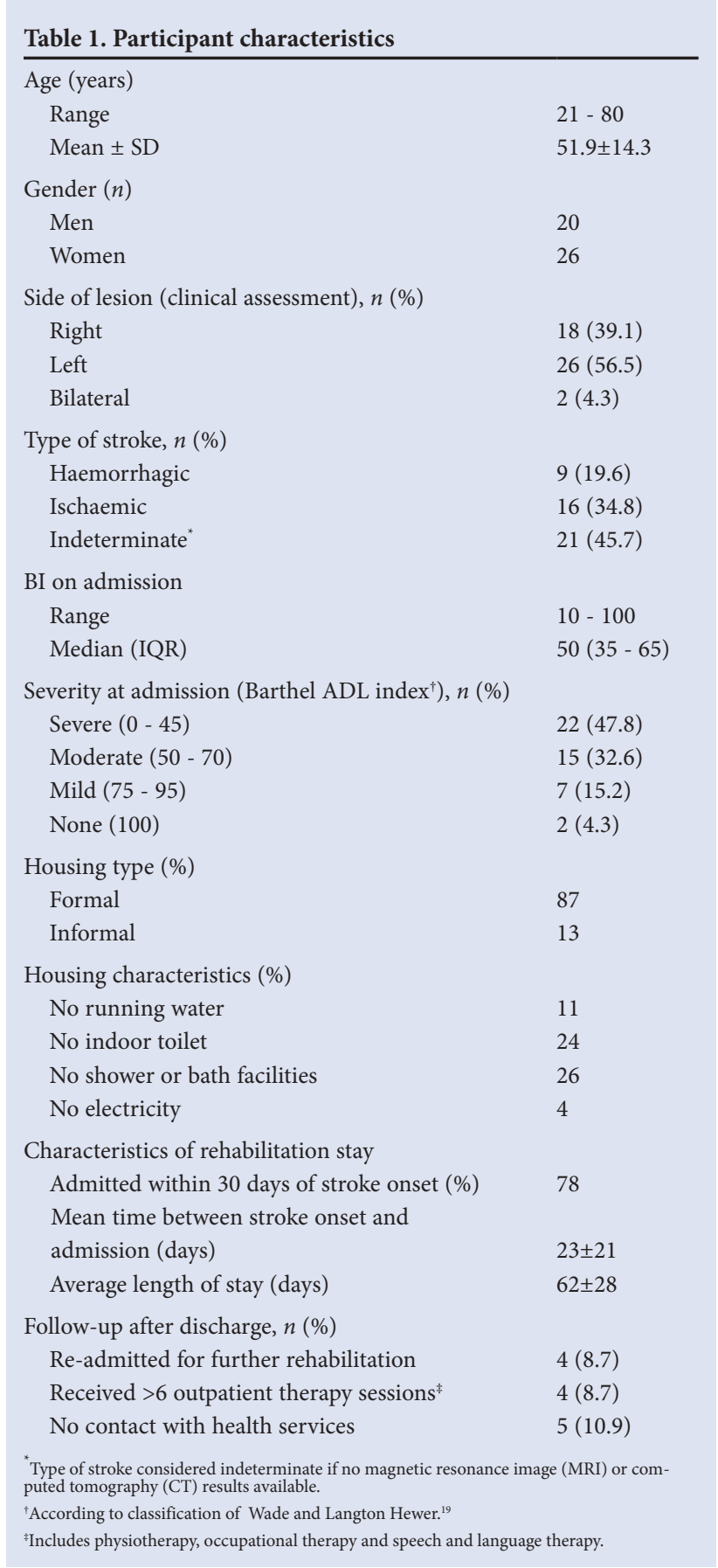

Table 2. Participants who performed items on the NEADL independently

\begin{tabular}{ll}
\hline & $\begin{array}{l}\text { Participants }(\boldsymbol{N}=\mathbf{4 6}) \\
\boldsymbol{n}(\%)\end{array}$ \\
\hline Mobility & $32(69.6)$ \\
Walk outside & $32(69.6)$ \\
Get in and out of car & $27(58.7)$ \\
Walk over uneven ground & $25(54.3)$ \\
Cross roads & $18(39.1)$ \\
Climb stairs & $16(34.8)$ \\
Travel on public transport & \\
Household & $46(100.0)$ \\
Feed yourself & $29(63.0)$ \\
Make a hot drink & $28(60.9)$ \\
Take hot drink from one room to another \\
Manage own money when out & $28(60.9)$ \\
Wash up & $26(56.5)$ \\
Make a hot snack & $22(47.8)$ \\
Wash small items of clothing & $19(41.3)$ \\
Do own housework & $18(39.1)$ \\
Wash a full load of clothes & $13(28.3)$ \\
Own shopping & $9(19.6)$ \\
Leisure & $37(80.4)$ \\
Use telephone & $33(71.7)$ \\
Read newspaper/book & $12(26.1)$ \\
Manage own garden & $12(26.1)$ \\
Go out socially & $8(17.4)$ \\
Write letter & $2(4.3)$ \\
Drive a car & \\
&
\end{tabular}

(next of kin could not be reached). Fifty-one were registered; 1 died, 1 was institutionalised, 1 could not be contacted, 1 declined consent, and 1 repeatedly did not arrive for scheduled assessments. Forty-six patients were thus assessed in the community at 6 months post stroke.

The mean interval of onset to admission was $23 \pm 21$ days; most (78.2\%) participants gained admission to rehabilitation within 30 days of stroke. Mean length of stay in rehabilitation was $62 \pm 28$ days. Many participants did not have any substantial follow-up after discharge (Table 1).

Fig. 1 shows the percentage of participants needing help with individual items of the BI at 6 months. Fourteen participants (30.4\%) were independent for all items $(\mathrm{BI}=100)$ and $20(43.5 \%)$ had mild disability (BI=75 - 95). The median BI was 90 (IQR 70 - 100).

Table 2 outlines the number and percentage of the cohort who performed NEADL activities independently. MRS scores (Table $3)$ indicated that $20 \%(n=9)$ of participants had severe disability (MRS=5) and the need for constant caregiver availability; 9\% $(n=4)$ could be left alone but needed assistance with basic needs such as toileting, hygiene and eating (MRS $=4)$; half the participants $(n=23)$ had moderate disability (MRS=3); $17 \%(n=8)$ could look after their own affairs but were not able to participate in all usual activities/ roles $(\mathrm{MRS}=2)$; and $4 \%(n=2)$ experienced no significant disability and could carry out all previous activities and duties $(\mathrm{MRS}=1)$. No participants were free of symptoms (MRS $=0$ ). 
Table 3. Scores according to the Modified Rankin Scale

\begin{tabular}{|c|c|c|}
\hline MRS & Description & $\begin{array}{l}\text { Participants }(N=46) \\
n(\%)\end{array}$ \\
\hline 5 & Severe disability: requires constant caregiver & $9(20)$ \\
\hline 4 & Needs assistance with basic needs but can be left alone & $4(9)$ \\
\hline 3 & $\begin{array}{l}\text { Moderate disability: independent in basic self-care, but needs assistance with meal preparation, } \\
\text { housework, shopping, financial management or travel }\end{array}$ & $23(50)$ \\
\hline 2 & Able to look after own affairs, not able to participate in all usual activities/roles & $8(17)$ \\
\hline 1 & No significant disability: able to carry out all previous activities/duties & $2(4)$ \\
\hline 0 & No symptoms & $0(0)$ \\
\hline
\end{tabular}

Table 4. Participants reporting changes in role since stroke according to the MRS structured interview responses $(N=46)$

\begin{tabular}{lllll}
\hline & $\begin{array}{l}\text { Unable } \\
\boldsymbol{n}(\%)\end{array}$ & $\begin{array}{l}\text { Reduced ability } \\
\boldsymbol{n}(\%)\end{array}$ & $\begin{array}{l}\text { No change } \\
\boldsymbol{n}(\%)\end{array}$ & $\begin{array}{l}\text { Not a previous role } \\
\boldsymbol{n}(\%)\end{array}$ \\
\hline Leisure and social life & $7(15.2)$ & $31(67.4)$ & $7(15.2)$ & $1(2.2)$ \\
Family responsibilities & $14(30.4)$ & $13(28.3)$ & $3(6.52)$ & $16(34.8)$ \\
Work/seeking work & $18(39.1)$ & $10(21.7)$ & $2(4.3)$ & $16(34.8)$
\end{tabular}

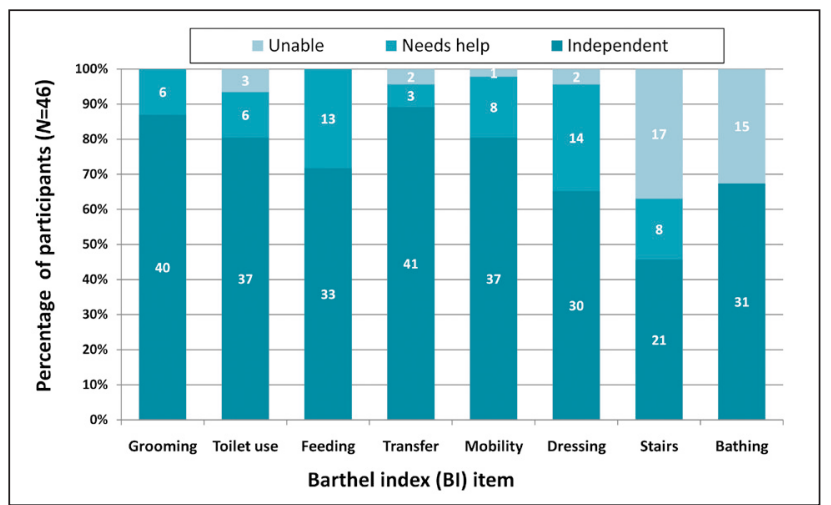

Fig. 1. Percentage of participants $(\mathrm{N}=46)$ needing help with individual items of the Barthel index at 6 months. Mobility categories of 'walks with help of one person' and 'wheelchair independent' have been condensed into 'needs help'. Transfer categories of 'major help' and 'minor help' have been condensed into 'needs help'.

In individual responses to questions addressing change in role (Table 4 ), $82.6 \%, 60.8 \%$ and $58.7 \%$ reported that they were unable, or had a reduced ability, to participate in social and leisure activities, work activities and family responsibilities, respectively. A further 37\% reported problems with relationships or feelings of isolation.

Two participants with severe language or cognitive deficits were excluded from the EQ-5D follow-up. Included participants $(N=44)$ reported problems in all quality of life domains, with the least problems reported in self-care $(30 \%)$. Ability to perform usual activities (61\%) was the most affected - 27\% reported severe problems, 59\% reported pain or discomfort, 50\% reported feelings of anxiety or depression, and 52\% reported problems with mobility. Visual analogue scores of perceived health state ranged from 30 to 100, with a median of 70.0 (IQR 57 - 85).

Data were available for the caregivers of 41 patients; 1 participant did not need a caregiver, and 4 caregivers failed to return the questionnaires. Fifty-six per cent of caregivers had CSI scores $\geq 7$, reflecting high levels of strain. ${ }^{18}$ Financial strain (65.9\%) and work adjustments (53.6\%) featured prominently.

\section{Discussion}

Stroke survivors undergoing intensive inpatient rehabilitation at a regional specialist centre in the Western Cape were studied. Their young average age was striking; persons of this age would be expected to be economically active and have dependents to support. Moreover, participants were largely from a low income bracket.

ADL outcomes were favourable with $73.9 \%$ of patients independent in ADL or having minimal disability, defined by BI scores of $75-100 .^{19}$ Thirty per cent of participants had a BI score of 100 at 6 months compared with $20 \%$ of stroke survivors in the $\mathrm{CHCC}$ cohort receiving less intensive rehabilitation. ${ }^{7}$ Moreover, the median BI of 90 at 6 months in our study is similar to European stroke units in the CERISE study (median range $85-100$ ). ${ }^{20}$ However, differences in age and severity profiles between cohorts are likely to complicate comparisons. Assistance was mostly required for stair-climbing, dressing and bathing - as in local and international studies., ${ }^{2,7}$

Although most participants were independent in ADL, results of the NEADL showed that many required assistance with instrumental activities required for living at home, such as preparing hot drinks and snacks, washing up, washing clothes and doing the housework. This implies a burden of care on family members and, for some, constitutes a loss of role.

According to the NEADL, few participants had independently participated in activities outside the home, such as doing their own shopping and going out unassisted socially.

In terms of travel ability, $34.8 \%$ had used public transport and $69.6 \%$ had walked outside and got in and out of a car. However, given the low average income, few participants would own cars, making inability to travel by public transport particularly serious. Stroke survivors experience difficulties in using public transport in SA. ${ }^{78}$ Independent functional walking within the community is another common difficulty, owing to residual impairments. Although $80 \%$ of all participants could walk independently indoors (mobility item in the BI), this does not imply ability to achieve safe walking in the community. Few participants had performed the more demanding items of crossing roads $(54.3 \%)$, walking over uneven ground $(58.7 \%)$ or climbing stairs $(39.1 \%)$. Difficulties in community mobility are exacerbated by environmental barriers such 
as unfavourable terrain and local public transport that are not geared to persons with disability. The NEADL identifies actual performance rather than ability and does not distinguish between limitations of innate disability and environmental restrictions. Dependence for community mobility increases the burden on caregivers and limits opportunities for community participation. The lack of suitable and affordable public transport for persons with stroke, impacts the access to participation in activities outside the home and to healthcare. Transport for persons with disabilities must be addressed at government level.

Three features of the MRS results warrant highlighting: (i) severe disability (MRS of 4 or 5) was experienced by $29 \%$ of stroke survivors, with $20 \%$ requiring a caregiver, typically a family member, to be present at all times; (ii) about half of the participants were independent in essential ADL but required assistance from family members to sustain community living $(\mathrm{MRS}=3$ ); and (iii) a high proportion reported a loss of role including many with only mild levels of disability.

Visual analogue scores pertaining to health-related quality of life on the EQ-5D were similar to those of the CERISE project (median of 70 v. $60-70$ in the European centres). ${ }^{20}$ It is clinically important that $59 \%$ of participants reported pain and discomfort and 50\% reported feelings of anxiety and depression, as these are potentially modifiable.

The caregiver strain of $58 \%$ was similar to that of another study at the same facility, but was higher than in international studies. ${ }^{8}$ Stroke survivors in developed countries received more therapies, nursing services, contact with doctors and psychologists after discharge, and severe stroke patients were more likely to be institutionalised at 6 months after stroke. ${ }^{20}$ Because SA has few suitable residential facilities, patients with severe disabilities are discharged into the care of families, often without support and follow-up. ${ }^{8}$ Lack of community support services and financial pressures contribute to higher caregiver strain and to a double loss of income as family members must give up work to care for the stroke survivor. ${ }^{8}$

Levels of community participation reflect environmental factors and the level of impairment of the stroke patient. Given their low incomes, lack of financial resources is an additional barrier to functioning. Furthermore, low-cost housing features such as outdoor toilets $(23 \%)$ and no running water inside (11\%) affect the complexity of ADL for tasks such as toileting, and extended ADL such as washing up and food preparation.

In terms of rehabilitation, most participants were admitted within 30 days of stroke and length of hospital stay was similar to or longer than that of international studies. ${ }^{9}$ However, participants received little input after discharge and $10 \%$ had no further contact with healthcare services. Consequently, potentially modifiable problems such as pain and depression were not identified and support of patients and caregivers over the transition after discharge was lacking. Intervention after discharge improves performance in extended ADL and community mobility. ${ }^{20,21}$

Four (8.7\%) participants were re-admitted for a second rehabilitation stay to optimise functioning. While the study did not permit further analysis, short re-admissions at a later stage of recovery may facilitate reintegration and improved community participation; the cost-and-benefit thereof requires further investigation. Resources limit what can be provided realistically in the healthcare system of SA; research is needed to identify interventions in the community after discharge that can make a difference at the least cost, identify patients most likely to benefit, and assess whether targeted programmes may improve specific aspects of functioning.

As the study reported functioning in stroke survivors admitted to a single rehabilitation unit in Cape Town, care should be taken with generalising the results.

Acknowledgements. The authors thank the Medical Research Council of South Africa and South African Society of Physiotherapy for funding, and the Katholieke Universiteit Leuven, Belgium, for funding and academic support. Anthea Rhoda (University of the Western Cape, SA) is acknowledged for assistance with validation of instrumentation.

\section{References}

1. Connor M, Bryer A. Stroke in South Africa. Cape Town: Medical Research Council, 2006. http://www. mrc.ac.za/chronic/cdlchapter 14.pdf (accessed 23 September 2006),

2. Wilkinson PR, Wolfe C, Warburton FB, et al. A long-term follow-up of stroke patients. Stroke 1997; 28(3):507-512. [http://dx.doi.org/10.1161/01.STR.28.3.507]

3. Desrosiers J, Rochette A, Noreau L, et al. Long-term changes in participation after stroke. Top Stroke Rehabil 2006;13(4):86-97. [http://dx.doi.org/10.1310/tsr1304-86]

4. Ustun T, Chatterji S, Bickenbach J, Kostanjsek N, Schneider M. The international classification of functioning, disability and health: A new tool for understanding disability and health. Disabil Rehabil 2003;25(11-12):565-571. [http://dx.doi.org/10.1080/0963828031000137063]

5. The SASPI Project Team. Prevalence of stroke survivors in rural South Africa: results from the Southern Africa Stroke Prevention Initiative (SASPI) Agincourt field site. Stroke 2004;35:627-632. [http://dx.doi.org/10.1161/01.STR.0000117096.61838.C7]

6. Wasserman S, de Villiers L, Bryer A. Community-based care of stroke patients in a rural African setting. S Afr Med J 2009;99:579-583

7. Rhoda A, Mpofu R, De Weerdt W. Activity limitations of patients with stroke attending out-patient facilities in the Western Cape, South Africa. SA Journal of Physiotherapy 2011;67(2):16-22.

8. Hassan S, Visagie S, Mji G. Strain experienced by caregivers of stroke survivors in the Western Cape. SA Journal of Physiotherapy 2011;67(2):4-8.

9. De Wit L, Putman K, Schuback B, et al. Motor and functional recovery after stroke: A comparison De Wit L, Putman K, Schuback B, et al. Motor and functional recovery after stroke: A comparison
of 4 European rehabilitation centers. Stroke 2007;38:2101-2107. [http://dx.doi.org/10.1161/ STROKEAHA.107.482869]

10. World Health Organization. The World Health Organization MONICA Project. J Clin Epidemiol 1988;41:105-114.

11. Sulter G, Steen C, De Keyser J. Use of the Barthel Index and Modified Rankin Scale in Acute Stroke Trials. Stroke 1999;30:1538-1541. [http://dx.doi.org/10.1161/01.STR.30.8.1538]

12. Nouri F, Lincoln N. An extended activities of daily living index for stroke patients. Clin Rehabil 1987;1:301-305.

13. van Swieten J, Koudstaal P, Visser M, Schouten H, van Gijn J. Interobserver agreement for the assessment of handicap in stroke patients. Stroke 1988;19:604-607. [http://dx.doi.org/10.1161/ 01.STR.19.5.604]

14. Wilson JTL, Hareendran A, Grant M, et al. Improving the assessment of outcomes in stroke: use of a structured interview to assign grades on the Modified Rankin scale. Stroke 2002;33:2243-2246. [http:// dx.doi.org/10.1161/01.STR.0000027437.22450.BD]

15. Dorman PJ, Waddell F, Slattery J Dennis M, Sandercock P. Is the EuroQol a Valid Measure of HealthDorman PJ, Waddell F, Slattery J, Dennis M, Sandercock P. Is the EuroQol a Valid Measure of Health-
Related Quality of Life after Stroke? Stroke 1997b;28:1876-1882. [http://dx.doi.org/10.1161/01. Related Quality

6. Robinson B. Validation of a Caregiver Strain Index. Journal of Gerontology 1983;38:344-348.

17. Dorman P, Waddell F, Slattery J, Dennis M, Sandercock P. Are proxy assessments of health status after stroke with the EuroQol questionnaire feasible, accurate, and unbiased? Stroke 1997;28(10):1883-1887. [http://dx.doi.org/10.1161/01.STR.28.10.1883]

18. Blake H, Lincoln N, Clarke D. Caregiver strain in spouses of stroke patients. Clin Rehabil 2003;17:312317. [http://dx.doi.org/10.1191/0269215503cr613oa]

19. Wade D, Langton Hewer R. Functional abilities after stroke: measurement, natural history and prognosis. J Neurol Neurosurg Psychiatry 1987;50:177-182.

20. Putman $\mathrm{K}$, Wit L, Schupp W, et al. Variations in follow-up services after inpatient stroke rehabilitation: A multicentre study. Journal of Rehabilitation Medicine 2009;41(8):646-653.

21. Logan P, Gladman J, Avery A, Walker M, Dyas J, Groom L. Randomised controlled trial of an occupational therapy intervention to increase outdoor mobility after stroke. BMI 2004:329(7479):1372. [http://dx.doi.org/10.1136/bmj.38264.679560.8F]

Accepted 9 February 2012. 